\title{
BMJ Open Effectiveness of cognitive behavioural therapy on quality of life in patients with prostate cancer after androgen deprivation therapy: a protocol for systematic review and meta-analysis
}

\author{
Fan Yuan (ㄷ, , Degui Chang, ${ }^{1}$ Mingyi Jing, ${ }^{2}$ Baohua Zhu, ${ }^{2}$ Yaodong You ${ }^{1}$
}

To cite: Yuan F, Chang D, Jing $\mathrm{M}$, et al. Effectiveness of cognitive behavioural therapy on quality of life in patients with prostate cancer after androgen deprivation therapy: a protocol for systematic review and meta-analysis. BMJ Open 2021;11:e049314. doi:10.1136/ bmjopen-2021-049314

- Prepublication history for this paper is available online. To view these files, please visit the journal online (http://dx.doi. org/10.1136/bmjopen-2021049314).

Received 21 January 2021 Accepted 19 0ctober 2021

Check for updates

(c) Author(s) (or their employer(s)) 2021. Re-use permitted under CC BY-NC. No commercial re-use. See rights and permissions. Published by BMJ.

${ }^{1}$ Department of Urology and Andrology, Hospital of Chengdu University of Traditional Chinese Medicine, Chengdu, Sichuan,

China

${ }^{2}$ Department of Dermatology, Hospital of Chengdu University of Traditional Chinese Medicine, Chengdu, Sichuan, China

Correspondence to

Dr Yaodong You;

yyd110@163.com

\section{ABSTRACT}

Introduction Prostate cancer (PCa), as a malignant tumour with rapid development in recent years, significantly affects men's health, work, life and economy. Androgen deprivation therapy (ADT) plays an important role in the treatment of $\mathrm{PCa}$ and can be used as a complementary therapy in the late stage of castrationresistant prostate cancer. Though ADT targeting PCa shows an effective therapeutic effect, the underlying side effects (cognitive disorder, hot flashes, a decrease in sexuality) cannot be ignored. At present, cognitive behavioural therapy (CBT) has been widely used for patients with $\mathrm{PCa}$ after ADT due to its confirmed efficacy, fewer side effects and lower economic burden. However, the effectiveness of $\mathrm{CBT}$ for patients with $\mathrm{PCa}$ after $\mathrm{ADT}$ is still controversial. Therefore, we will conduct a systematic review and metaanalysis of the effectiveness of CBT for patients with $\mathrm{PCa}$ after ADT.

Methods and analysis Literatures will be searched from establishment of the database to 31 May 2021 with the language restrictions of English and Chinese in eight online databases (PubMed, Embase, the Web of Science, Cochrane Library, VIP, CNKI, CBM, and WAN FANG). This study will include RCTs that performed CBT as the main method of the experimental group for patients with $\mathrm{PCa}$ after ADT. Two or more reviewers will independently conduct the selection of studies, data extraction and data analysis. The risk ratios with $95 \%$ Cls will be used to present the data synthesis result of dichotomous data, while weighted mean differences or standardised mean differences with $95 \%$ Cls will be used to present the data synthesis result of continuous data. Meanwhile, evidence quality of outcome will be assessed by using the Grading of Recommendations Assessment, Development and Evaluation method. Stata V.13.0 and Review Manager software V.5.3 will be used for analysis and synthesis.

Ethics and dissemination This protocol is a second study based on a completed randomised controlled study. Thus, ethical approval is not required, and no additional data are available.

OSF registration number DOI 10.17605/OSF.I0/FUVEA
Strengths and limitations of this study

- This is a protocol for the first systematic review and meta-analysis of the existing prospective, longitudinal evidence on cognitive behavioural therapy (CBT) for patients with prostate cancer (PCa) after androgen deprivation therapy (ADT).

- This protocol will strictly adhere to the recommendations of the Cochrane handbook for systematic reviews of interventions.

- We predict that the pooled effects may be influenced by high statistical heterogeneity.

- We will use the Grading of Recommendations Assessment, Development and Evaluation system to assess evidence quality of outcomes, which would help clinicians and patients decide whether or not to choose CBT for treating PCa after ADT.

- A potential limitation of this protocol may be the lack of sufficient evidences on the intervention of CBT on the quality of life of patients with PCa after androgen deprivation treatment, and there may be heterogeneity in available studies.

\section{INTRODUCTION}

Prostate cancer $(\mathrm{PCa})$ is a malignant tumour occurring in the prostate, and its incidence is gradually increasing. Studies showed that PCa was the second most frequently diagnosed cancer and the fifth leading cause of cancer mortality among men worldwide in $2012 .{ }^{1}$ The incidence and mortality of PCa had international variation. ${ }^{2}$ Recent studies suggest that the incidence and mortality of PCa are lower in Asia, but the regions with high incidence rates are Australia/New Zealand, northern America, and western and northern Europe. ${ }^{3}$ These variations may be caused by the regional differences in the diagnosis of PCa through PSA-based screening, the availability of treatment and lifestyle. ${ }^{45}$ However, the cause of PCa is not clear until now. The current consensus is that older age, ethnicity 
and genetic predisposition are the specific risk factors for PCa. ${ }^{6}$ Besides, unhealthy lifestyle is also a risk factor for PCa, such as obesity, diet high in calcium, dairy products. $^{7-9}$ The current treatments for PCa include: radical prostatectomy, endocrine therapy, external beam radiotherapy, plesioradiotherapy (both high and low doses), active surveillance and watchful waiting, cryotherapy, high intensity focused ultrasound, and so on. ${ }^{10-12}$

Androgen deprivation therapy (ADT), which is considered as one of endocrine therapies, is widely used in the treatment of patients with PCa, mainly including bilateral orchiectomy and luteinising hormone-releasing hormone (LHRH) agonists or antagonists, and LHRH agonists are the most commonly used at present. ${ }^{13}$ ADT can significantly prolong the life of patients with metastatic PCa in the earliest stage. ${ }^{14}$ However, with the continuation of ADT, problems such as drug-induced gonadal function decline and adverse reactions of treatment become more obvious. ${ }^{15} \mathrm{PCa}$ is a great psychological burden for patients. Hormonal changes caused by ADT make the situation worse. Studies have shown that about a third of patients will have severe anxiety and even suicidal tendencies in the first 12 months after diagnosis of metastatic disease. ${ }^{16}$ In addition, an RCT showed that half of the patients experienced a decline in cognitive abilities after 6 months of ADT. ${ }^{16}{ }^{17}$ Another study has also confirmed that the cognitive abilities of patients in the ADT group, especially memory, attention and information processing, are significantly lower than that of the non-ADT group and the healthy group. ${ }^{18}$ ADT inhibits the growth of cancer cells by reducing the concentration of androgens in the body and inhibiting the conversion of testosterone to dihydrotestosterone. However, with the decrease in androgen level, patients will show symptoms of hypogonadism, such as hot flashes, ${ }^{19}$ anxiety, ${ }^{19}$ a decrease in sexuality ${ }^{20}$ and even the occurrence of male breast cancer after antiandrogenic treatment. ${ }^{20}$ Besides, ADT can also lead to osteoporosis, ${ }^{21}{ }^{22}$ cardiovascular disease, ${ }^{2324}$ metabolic disorders ${ }^{2325}$ and other systemic diseases. The economic burden of other systemic diseases caused by ADT including the direct and indirect costs is substantial and significant.

Cognitive behavioural therapy (CBT, including psychological interventions) has been proven to be effective in reducing negative effects and improving the quality of life of patients with cancer. Studies have shown that cancer survivors have anxiety, depression and poor quality of life, ${ }^{26}$ and the severity of symptoms is associated with prolonged treatment cycles and increased use of medical resources. ${ }^{27} \mathrm{CBT}$ can relieve anxiety and depression in patients with cancer to a great extent, ${ }^{28}$ and it can also effectively treat persistent fatigue and insomnia caused by cancer. ${ }^{29}{ }^{30}$ In addition, a randomised controlled trial (RCT) showed that the quality of life in patients with PCa (Most of them non-Hispanic whites) was improved significantly after 10 weeks of cognitive behavioural stress management (awareness of disease, change of mood, communication with medical staffs, etc). ${ }^{31}$ Other studies have shown that psychoeducational interventions can improve the coping skills of patients with PCa and improve their sexual function to a some extent. ${ }^{32}$

Therefore, it is necessary to assess the issue and design this systematic review and meta-analysis to determine the effectiveness of CBT for patients with PCa after ADT based on the latest evidence.

\section{METHODS}

We will formulate this protocol based on the standard of 'Preferred Reporting Items for Systematic Review and Meta-analysis Protocols (PRISMA-P) ${ }^{33-35}$ And this study will be carried out on 1 June 2021.

\section{Patient and public involvement}

No patient and public were involved.

\section{Inclusion criteria}

Types of studies

Our research will include RCTs which access the effectiveness of CBT for patients with PCa after ADT.

\section{Types of patients}

Our objective population will be patients with PCa owing to the decrease in quality of life after ADT. The diagnostic criteria will be based on the prostate cancer-related quality of life (PCa-QoL) Scale. ${ }^{36}$

\section{Types of intervention}

CBT, including mindfulness-based cognitive therapy, ${ }^{37}$ psychological guidance, stress management and cognitive couple therapy, and so on, is defined as the treatment group. No treatment can be provided for the control groups.

\section{Types of outcome measures}

\section{Primary outcomes}

The primary outcome is the PCa-QoL scales. ${ }^{37}$ The PCaQoL Scale has two main components which including 11 subindex scales. Seven were generically relevant to patients with PCa: urinary control, sexual intimacy, sexual confidence, marital affection, masculine self-esteem, health worry and PSA concern. Four scales were specific to the treatment experience: perceived cancer control, quality of treatment decision making, regret of treatment choice and cancer-related outlook.

\section{Secondary outcomes}

The secondary outcomes mainly include the following aspects: the Hospital Anxiety and Depression Scale, ${ }^{38}$ Cancer Worry Scale, ${ }^{39}$ IIEF- $5,{ }^{40} 41$ recurrence rate.

\section{Exclusion criteria}

1. Grey literature such as animal experiments, mechanism of disease, case reports, systematic review, metaanalysis and meeting abstracts will be excluded. 


\section{Box 1 The search strategy of the Cochrane Library}

-\#1 MeSH descriptor: ((Prostatic Neoplasms)) explode all trees

- \#2 ('Prostatic Neoplasms'):ti,ab,kw OR ('Prostate Neoplasm'):ti,ab,kw OR ('Prostatic Neoplasm'):ti,ab,kw

> \#3 ('Prostate Cancer') :ti,ab,kw OR ('Prostatic Cancer*):ti,ab,kw OR ('Cancer of the Prostate'):ti,ab,kw OR ('PCa'):ti,ab,kw

- \#4 \#1 OR \#2 OR \#3

- \#5 MeSH descriptor: ((Cognitive Behavior Therapycognitive-behavioral therapy)) explode all trees

- \#6 ('Cognitive BehaviorBehaviour Therap*):ti,ab,kw OR ('Therapy, Cognitive BehaviorBehaviour'):ti,ab,kw OR ('BehavioralBehavioural Therapies, Cognitive'):ti,ab,kw OR ('CBT'):ti,ab,kw OR ('bCBT'):ti,ab,kw

- \#7 ('Therapies, Cognitive Behavio*'):ti,ab,kw OR ('Cognition Therap*'):ti,ab,kw OR ('Cognitive Psychotherapy'):ti,ab,kw

- \#8 ('Mind Body Medicine'):ti,ab,kw OR ('Mind-Body Therapies'):ti,ab,kw OR ('Mindful cognitive therapy'):ti,ab,kw OR ('MBCT'):ti,ab,kw OR ('mindfulness-based interventions'):ti,ab,kw

- \#9 ('Couples therapy'):ti,ab,kw OR ('Cognitive Couple Therapy'):ti,ab,kw OR ('stress management '):ti,ab,kw

- \#10 \#5 OR \#6 OR \#7 OR \#8 OR \#9

-\#11 MeSH descriptor: ((Randomised controlled trial)) explode all trees

- \#12 ('RandomizedRandomised controlled trial'):ti,ab,kw OR ('RandomizedRandomised'):ti,ab,kw OR ('controlled'):ti,ab,kw

\#13 \#11 OR \#12

\#14 \#4 AND \#10 AND \#13

2. The exclusion criteria for this study will be persons receiving treatment other than ADT and suffering from severe cognitive or psychiatric problems.

3. The patients in the control group were treated with any form of CBT.

4. Considering the longer treatment period of CBT, studies with treatment time less than 4 weeks will be excluded.

5. Studies from which raw data cannot be extracted and studies of which the authors cannot be contacted will be excluded.

\section{Search methods for identification of studies}

\section{Electronic searches}

English and Chinese databases will be searched from inception to 31 May 2021, including PubMed, Embase, Web of Science, Cochrane Library, VIP, China National Knowledge Infrastructure (CNKI), Chinese Biomedical Literature Database (CBM) and Wan Fang Data Knowledge Service Platform (WAN FANG). The key search terms of the study include 'Prostatic Neoplasms', 'PCa', 'Prostatic Cancer', 'Cancer of the Prostate', 'cognitivebehavioral therapy', 'Therapy, Cognitive Behaviour', 'Therapy, Cognitive Behaviour', 'Mind-Body Therapies', 'mindfulness-based intervention', 'Cognitive Couple Therapy', 'CBT', 'bCBT' and 'Randomised controlled trial'. The search strategy of the Cochrane Library is presented in box 1 .

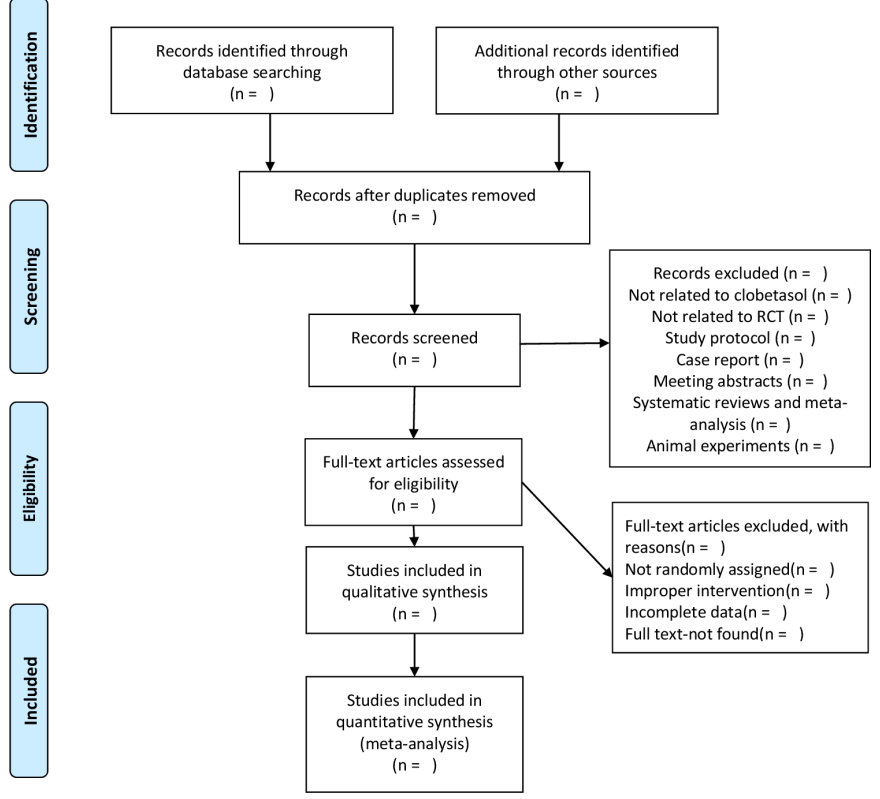

Figure 1 Process of study screening (adapted from Moher et al [51]). RCT, randomised controlled trial.

\section{Searching other resources}

We will search the National Institutes of Health clinical registry Clinical Trials, International Clinical Trials Registry Platform, Australian New Zealand Clinical Trials Registry and Chinese clinical registry to find the unpublished or ongoing trial data. Additionally, Interlibrary Loan and Baidu academic will be used to assist in the acquisition of full-text documents.

\section{Selection and management of studies}

All relevant references were selected, downloaded and uploaded in EndNote software. First, duplicates will be automatically deleted by EndNote(X8) software. Second, two researchers will filter out ineligible articles by title and abstracts based on the criterion of inclusion and exclusion. Moreover, they will read full texts if the studies cannot be screened only though the title and abstracts. Lastly, two reviewers cross-check after screening. If an inconsistent opinion exists, they will resolve it through discussion. Otherwise, a third researcher will make the judgement. The process and results of the studies' selection will be presented in a flow chart (figure 1).

\section{Data extraction}

Two reviewers independently extract data from the included studies based on the Cochrane Handbook 5.2.0 guidelines after literature screening. ${ }^{35}$ Data will include the following information:

1. Study information: author, country, journal, publication date.

2. Characteristics of patients: average age, numbers of patients, race, region, scores of outcomes at baseline and follow-up.

3. Intervening measure: type, duration and frequency of CBT. 
4. Methodological Information: information was extracted from six aspects including: random sequence generation, allocation concealment, blind method, result data integrity, selective reporting and other biases. The quality of the information will be assessed with standards of the Cochrane Handbook 5.2.0 which be graded into 'low risk', 'medium risk' and 'high risk' levels.

\section{Data analysis}

RevMan V.5.3 and Stata V.13.0 software will be used to analyse all data. Continuous variables such as the scale of $\mathrm{PCa}-\mathrm{QoL}$ and dichotomous variables such as the recurrence rate will be represented as weighted mean difference and relative risk with 95\% CI separately. The results of data analysis were considered statistically significant at a value of $\mathrm{p}<0.05$.

\section{Heterogeneity test and subgroup analysis}

The application of $Q$ and $\mathrm{I}^{2}$ statistics can detect both the existence and degree of heterogeneity. $Q$ test is obviously affected by the number of included studies, while $\mathrm{I}^{2}$ statistics will not change with the number of studies, and its results of heterogeneity test are more stable. When the value of $\mathrm{I}^{2}=0$, it indicates that the variation between studies is only caused by sampling error; when $0.25<$ $\mathrm{I}^{2}<0.5$, it is considered that there is moderate heterogeneity; when $\mathrm{I}^{2}>0.5$, high heterogeneity is identified. Meanwhile, the treatment method of heterogeneity is based on the values of $\mathrm{I}^{2}$ and $\chi^{2}$ statistics (expressed by $\mathrm{p}$ value). When $\mathrm{I}^{2}<50 \%$ and $\mathrm{p}>0.10$, a fixed-effect model was used; when $\mathrm{I}^{2} \geq 50 \%$ or $\mathrm{p}<0.10$, a random-effect model was used. It is necessary to find sources of heterogeneity through meta-regression, subgroup meta-analyses and sensitivity analysis. We will consider $\mathrm{I}^{2} \geq 50 \%$ or $\mathrm{p}<0.1$ as substantial heterogeneity, which will be further studied by subgroup analyses. Subgroup analyses will be carried out for the different interventions (eg, mindfulness-based intervention, cognitive couple therapy), length of treatment, and duration and frequency at baseline. Moreover, we will conduct subgroup analysis according to the participants' characteristics such as age and phase (advanced or not), to explore which group benefited the most.

\section{Sensitivity analysis}

Sensitivity analysis is mainly used to evaluate the reliability of the meta-analysis results. We will determine the stability of meta-analysis results by means of excluding studies with small sample sizes or low evidence. The results of metaanalysis can be accepted if the outcomes have no change after sensitivity analysis. Otherwise, we will be cautious with the results.

\section{Assessment of publication bias}

It is necessary to use an inverted funnel plot to evaluate publication bias when more than 10 studies are included. Publication bias depends on whether the funnel plot is symmetrical. If the number of studies is less than 10, Egger's linear regression method would be used to analyse the risk of bias. We can prevent publication bias though establishing a trial registration system, changing the editing and reviewing strategy, and so on.

\section{Grading the quality of evidence}

The development of clinical practice guidelines depend on the acquisition of scientific evidence. So, the quality of evidence will be classified into high, moderate, low or very low based on the Grading of Recommendations Assessment, Development and Evaluation guidelines system. ${ }^{42}$

\section{ETHICS AND DISSEMINATION}

Ethical approval is not required since this study is a secondary analysis of existing literature. Also, we hope to publish our study in a peer-reviewed journal or present at relevant conferences for clinical doctors and patients.

\section{DISCUSSION}

The quality of life of patients with PCa after ADT may be reduced due to maladjustment of androgen levels or fear of cancer recurrence (FCR). CBT, which is a nondrug therapy psychological intervention, can improve patients' confidence in the treatment of diseases by correcting patients' cognitive bias, enhancing patients' beliefs and changing attitudes towards their own problems from irrational knowledge to rational knowledge. ${ }^{43}$ In addition, CBT effectively improves the quality of life in patients with cancer, with anxiety, depression, ${ }^{44}$ fatigue, ${ }^{45}$ insomnia, ${ }^{30}{ }^{46}$ hot flashes, ${ }^{47}$ sexual dysfunction, ${ }^{48}$ and so on. In an RCT of blended cognitive behavioural therapy (bCBT) to reduce the severity of FCR in cancer survivors, FCR scores were significantly lower in the bCBT group than in the care as usual group. ${ }^{49}$ Another study has found that parents of children with cancer often experienced mental pressure, but post-traumatic stress symptoms, depression and anxiety were significantly improved by providing CBT training to parents. ${ }^{50}$ Therefore, we plan to study the effectiveness of CBT in patients with PCa after ADT. If the results of the study prove that CBT is an effective treatment for patients with PCa after ADT, it will improve the quality of life in patients with PCa after ADT and save on medical expenses.

This systematic review has the following limitations: (1) In order to ensure the quality of research, we have formulated strict standards for admission, which can lead to a limited number of studies. Therefore, it is recommended to increase the corresponding RCTs. (2) Because of language limitations, this study only included literature in Chinese and English, and does not include any literature in other languages. We should consider including literature in other languages. (3) The quality of original trials will affect the quality of the pooled effects. In order to demonstrate these effects on our assessment and promote the use of evidence in practice, we will assess the quality of evidence for the primary outcomes.

Contributors FY and YY contributed to the design of the research and concept of the review. FY and BZ developed the search strategy. MJ and BZ will search, 
select and identify the studies included, and extract data independently. DC will be the third reviewer for study selection and data extraction. FY and MJ drafted the manuscript. All authors have approved the publication of this protocol.

Funding This study is supported by the National Natural Science Foundation of China (Grant No.81973866/81503589).

\section{Competing interests None declared.}

Patient and public involvement Patients and/or the public were not involved in the design, or conduct, or reporting, or dissemination plans of this research.

Patient consent for publication Not applicable.

Provenance and peer review Not commissioned; externally peer reviewed.

Open access This is an open access article distributed in accordance with the Creative Commons Attribution Non Commercial (CC BY-NC 4.0) license, which permits others to distribute, remix, adapt, build upon this work non-commercially, and license their derivative works on different terms, provided the original work is properly cited, appropriate credit is given, any changes made indicated, and the use is non-commercial. See: http://creativecommons.org/licenses/by-nc/4.0/.

\section{ORCID iD}

Fan Yuan http://orcid.org/0000-0001-6377-8424

\section{REFERENCES}

1 Kimura T, Egawa S. Epidemiology of prostate cancer in Asian countries. Int J Urol 2018;25:524-31.

2 Center MM, Jemal A, Lortet-Tieulent J, et al. International variation in prostate cancer incidence and mortality rates. Eur Urol 2012;61:1079-92.

3 Culp MB, Soerjomataram I, Efstathiou JA, et al. Recent global patterns in prostate cancer incidence and mortality rates. Eur Urol 2020;77:38-52.

4 Tonon L, Fromont G, Boyault S, et al. Mutational profile of aggressive, localised prostate cancer from African Caribbean men versus European ancestry men. Eur Urol 2019;75:11-15.

5 Tikkinen KAO, Dahm P, Lytvyn L, et al. Prostate cancer screening with prostate-specific antigen (PSA) test: a clinical practice guideline. BMJ 2018;362:k3581.

6 Platz EA, Giovannucci E. Prostate cancer. In: Cancer epidemiology and prevention. Oxford University Press, 2006: 1128-50.

7 Allott EH, Masko EM, Freedland SJ. Obesity and prostate cancer: weighing the evidence. Eur Urol 2013;63:800-9.

8 Renehan AG, Tyson M, Egger M, et al. Body-Mass index and incidence of cancer: a systematic review and meta-analysis of prospective observational studies. The Lancet 2008;371:569-78.

9 Maclnnis RJ, English DR. Body size and composition and prostate cancer risk: systematic review and meta-regression analysis. Cancer Causes Control 2006;17:989-1003.

10 Cornford P, Bellmunt J, Bolla M, et al. EAU-ESTRO-SIOG guidelines on prostate cancer. Part II: treatment of relapsing, metastatic, and castration-resistant prostate cancer. Eur Urol 2017;71:630-42.

11 Mottet N, Bellmunt J, Bolla M, et al. EAU-ESTRO-SIOG guidelines on prostate cancer. Part 1: screening, diagnosis, and local treatment with curative intent. Eur Urol 2017;71:618-29.

12 Sanda MG, Cadeddu JA, Kirkby E, et al. Clinically localized prostate cancer: AUA/ASTRO/SUO guideline. Part II: recommended approaches and details of specific care options. J Urol 2018;199:990-7.

13 Wilke D, Patil N, Hollenhorst $\mathrm{H}$, et al. Testosterone suppression with luteinizing hormone-releasing hormone $(\mathrm{LHRH})$ agonists in patients receiving radiotherapy for prostate cancer. Pharmacotherapy 2018;38:327-33.

14 Heidenreich A, Bastian PJ, Bellmunt J. Guidelines on prostate cancer. Arnhem: European Association of Urology, 2012.

15 Rhee H, Gunter JH, Heathcote P, et al. Adverse effects of androgendeprivation therapy in prostate cancer and their management. BJU Int 2015;115:3-13.

16 Llorente MD, Burke M, Gregory GR, et al. Prostate cancer: a significant risk factor for late-life suicide. Am J Geriatr Psychiatry 2005;13:195-201.

17 Green HJ, Pakenham KI, Headley BC, et al. Altered cognitive function in men treated for prostate cancer with luteinizing hormonereleasing hormone analogues and cyproterone acetate: a randomized controlled trial. BJU Int 2002;90:427-32.

18 Yang J, Zhong F, Quu J, et al. Cognitive function in Chinese prostate cancer patients on androgen-deprivation therapy: a cross-sectional study. Asia Pac J Clin Oncol 2015;11:277-81.
19 Walker LM, Tran S, Robinson JW. Luteinizing hormone-releasing hormone agonists: a quick reference for prevalence rates of potential adverse effects. Clin Genitourin Cancer 2013;11:375-84.

20 Green HJ, Pakenham KI, Headley BC, et al. Quality of life compared during pharmacological treatments and clinical monitoring for nonlocalized prostate cancer: a randomized controlled trial. BJU Int 2004;93:975-9.

21 Wadhwa VK, Weston R, Mistry R, et al. Long-Term changes in bone mineral density and predicted fracture risk in patients receiving androgen-deprivation therapy for prostate cancer, with stratification of treatment based on presenting values. BJU Int 2009;104:800-5.

22 Hamilton EJ, Ghasem-Zadeh A, Gianatti E, et al. Structural decay of bone microarchitecture in men with prostate cancer treated with androgen deprivation therapy. J Clin Endocrinol Metab 2010;95:E456-63.

23 Keating NL, O'Malley AJ, Freedland SJ, et al. Does comorbidity influence the risk of myocardial infarction or diabetes during androgen-deprivation therapy for prostate cancer? Eur Urol 2013:64:159-66.

24 Levine GN, D'Amico AV, Berger P, et al. Androgen-Deprivation therapy in prostate cancer and cardiovascular risk: a science Advisory from the American heart association, American cancer Society, and American urological association: endorsed by the American Society for radiation oncology. Circulation 2010;121:833-40.

25 Smith MR, Finkelstein JS, McGovern FJ, et al. Changes in body composition during androgen deprivation therapy for prostate cancer. J Clin Endocrinol Metab 2002;87:599-603.

26 Mei Hsien CC, Wan Azman WA, Md Yusof M, et al. Discrepancy in patient-rated and oncologist-rated performance status on depression and anxiety in cancer: a prospective study protocol. BMJ Open 2012;2:e001799.

27 Prieto JM, Atala J, Blanch J, et al. Role of depression as a predictor of mortality among cancer patients after stem-cell transplantation. JCO 2005;23:6063-71.

28 Osborn RL, Demoncada AC, Feuerstein M. Psychosocial interventions for depression, anxiety, and quality of life in cancer survivors: meta-analyses. Int J Psychiatry Med 2006;36:13-34.

29 Gielissen MFM, Verhagen S, Witjes F, et al. Effects of cognitive behavior therapy in severely fatigued disease-free cancer patients compared with patients waiting for cognitive behavior therapy: a randomized controlled trial. J Clin Oncol 2006;24:4882-7.

30 Johnson JA, Rash JA, Campbell TS, et al. A systematic review and meta-analysis of randomized controlled trials of cognitive behavior therapy for insomnia (CBT-I) in cancer survivors. Sleep Med Rev 2016:27:20-8.

31 Penedo FJ, Dahn JR, Molton I, et al. Cognitive-Behavioral stress management improves stress-management skills and quality of life in men recovering from treatment of prostate carcinoma. Cancer 2004;100:192-200.

32 Mishel MH, Belyea M, Germino BB, et al. Helping patients with localized prostate carcinoma manage uncertainty and treatment side effects. Cancer 2002;94:1854-66.

33 Shamseer L, Moher D, Clarke M, et al. Preferred reporting items for systematic review and meta-analysis protocols (PRISMA-P) 2015 elaboration and explanation. BMJ 2015;349:g7647.

34 Stewart LA, Clarke M, Rovers M, et al. Preferred reporting items for a systematic review and meta-analysis of individual participant data. JAMA 2015;313:1657-65.

35 Higgins JPT, Green S, eds. Cochrane Handbook for Systematic Reviews of Interventions Version 5.2.0. The Cochrane Collaboration, 2017. www.cochrane-handbook.org

36 Clark JA, Bokhour BG, Inui TS, et al. Measuring Patients' perceptions of the outcomes of treatment for early prostate cancer. Med Care 2003;41:923-36

37 Piet J, Würtzen $\mathrm{H}$, Zachariae R. The effect of mindfulness-based therapy on symptoms of anxiety and depression in adult cancer patients and survivors: a systematic review and meta-analysis. $J$ Consult Clin Psychol 2012;80:1007-20.

38 Zigmond AS, Snaith RP. The hospital anxiety and depression scale. Acta Psychiatr Scand 1983;67:361-70.

39 Lebel S, Ozakinci G, Humphris G, et al. From normal response to clinical problem: definition and clinical features of fear of cancer recurrence. Support Care Cancer 2016;24:3265-8.

40 Hatzimouratidis K, Amar E, Eardley I, et al. Guidelines on male sexual dysfunction: erectile dysfunction and premature ejaculation. Eur Urol 2010;57:804-14.

41 Rosen RC, Riley A, Wagner G, et al. The International index of erectile function (IIEF): a multidimensional scale for assessment of erectile dysfunction. Urology 1997;49:822-30. 
42 Aguayo-Albasini JL, Flores-Pastor B, Soria-Aledo V. Grade system: classification of quality of evidence and strength of recommendation. Cir Esp 2014;92:82-8.

43 Stagl JM, Lechner SC, Carver CS, et al. A randomized controlled trial of cognitive-behavioral stress management in breast cancer: survival and recurrence at 11-year follow-up. Breast Cancer Res Treat 2015;154:319-28.

44 Shaffer KM, Kim Y, Carver CS. Physical and mental health trajectories of cancer patients and caregivers across the year postdiagnosis: a dyadic investigation. Psychol Health 2016;31:655-74.

45 Bower JE. Cancer-related fatigue--mechanisms, risk factors, and treatments. Nat Rev Clin Oncol 2014;11:597-609.

46 Qaseem A, Kansagara D, Forciea MA, et al. Management of chronic insomnia disorder in adults: a clinical practice guideline from the American College of physicians. Ann Intern Med 2016;165:125-39.

47 Yousaf O, Stefanopoulou E, Grunfeld EA, et al. A randomised controlled trial of a cognitive behavioural intervention for men who have hot flushes following prostate cancer treatment (MANCAN): trial protocol. BMC Cancer 2012;12:230.

48 Kang HS, Kim H-K, Park SM, et al. Online-based interventions for sexual health among individuals with cancer: a systematic review. BMC Health Serv Res 2018;18:167.

49 van de Wal M, Thewes B, Gielissen M, et al. Efficacy of blended cognitive behavior therapy for high fear of recurrence in breast, prostate, and colorectal cancer survivors: the sword study, a randomized controlled trial. J Clin Oncol 2017;35:2173-83.

50 Ljungman L, Cernvall M, Ghaderi A, et al. An open trial of individualized face-to-face cognitive behavior therapy for psychological distress in parents of children after end of treatment for childhood cancer including a cognitive behavioral conceptualization. PeerJ 2018;6:e4570.

51 Moher D, Liberati A, Tetzlaff J, et al. Preferred reporting items for systematic reviews and meta-analyses: the PRISMA statement. PLOS Med 2009;6:e1000097. 